\title{
Evaluación de diferentes granulometrías de calcio en la alimentación de gallinas ponedoras
}

\section{Evaluation of different calcium grain sizes in laying hens diet}

Fecha de recepción: 19 de diciembre de 2015

Fecha de aceptación: 5 de mayo de 2016

\author{
Rosa Angélica Sanmiguel-Plazas ${ }^{1}$ \\ Giovanny Rodrigo Mejía-Rojas ${ }^{2}$ \\ Luisa María Lozano-Covaleda ${ }^{3}$ \\ Román David Castañeda-Serrano ${ }^{4}$
}

\begin{abstract}
Resumen
En los últimos años, diversos estudios se han realizado para evaluar el efecto de la granulometría del calcio en la alimentación de gallinas ponedoras, dada su importancia en la eficiencia de la absorción de este mineral para la calcificación del huevo, especialmente en épocas en las que el ave disminuye el consumo de alimento; sin embargo, los resultados obtenidos son controvertidos. En el presente trabajo se propuso evaluar el efecto del tamaño de la partícula de calcio sobre el porcentaje de postura, el consumo de alimento, el peso del huevo y la conversión alimenticia. Se sometieron 120 gallinas ponedoras de la línea $\mathrm{Hy}$-line blanca de 90 semanas de edad, distribuidas al azar, y se aplicaron 4 tratamientos, cada uno con partículas de calcio de diferente tamaño: $\mathrm{T} 1=$ menor a $1 \mathrm{~mm}, \mathrm{~T} 2=3 \mathrm{~mm}, \mathrm{~T} 3=6 \mathrm{~mm}$ y T4=9 mm; cada tratamiento tuvo 10 repeticiones de 3 aves en cada unidad experimental. Hubo un efecto cuadrático para el porcentaje de postura entre los tratamientos a medida que aumentó el tamaño de partícula hasta el tratamiento T3, siendo el tratamiento T4 el que presentó menor porcentaje de postura y menor consumo de ración. Aunque no hubo diferencias significativas entre los tratamientos para el peso del huevo, la conversión alimenticia fue mejor en el tratamiento T4. Se concluye que es posible utilizar los tamaños de partícula de $3 \mathrm{~mm}$ y $6 \mathrm{~mm}$ sin afectar negativamente los parámetros de desempeño animal en gallinas ponedoras de más de 90 semanas de edad.
\end{abstract}

Palabras clave: alimentación de gallinas; avicultura; partícula de calcio.

1 M.Sc. Universidad Cooperativa de Colombia (Ibagué, Colombia). rosa.sanmiguel@campusucc.edu.co.

2 Universidad Cooperativa de Colombia (Ibagué, Colombia).

3 Universidad Cooperativa de Colombia (Ibagué, Colombia).

4 Ph.D. Universidad del Tolima (Ibagué, Colombia). rcastaneda@ut.edu.co. 


\begin{abstract}
The calcium grain size is important for absorption efficiency and calcification of the egg, especially when the bird's feeding decreases. In recent years, several studies have evaluated the effect of different calcium grain sizes on the feeding of laying hens; however, the results are controversial. This research evaluates the effect of particle size on the posture rate, feed intake, egg weight, and feed conversion. $120 \mathrm{Hy}$-line White 90 weeks old laying hens, randomly distributed, received 4 treatments, each one with different calcium particle size: $\mathrm{T} 1=$ less than $1 \mathrm{~mm}, \mathrm{~T} 2=3 \mathrm{~mm}, \mathrm{~T} 3=6 \mathrm{~mm}$, and $\mathrm{T} 4=9 \mathrm{~mm}$. Each treatment was repeated 10 times with 3 birds per experimental unit. The posture percentage showed a quadratic effect, with the percentage increasing as the particle size increased until T3; however, T4 was the treatment with the lowest posture percentage and feed intake. Although there were no significant differences between treatments for egg weight, feed conversion had the lowest value with T4. In conclusion, particle sizes of $3 \mathrm{~mm}$ and $6 \mathrm{~mm}$ have no adverse effects on animal performance parameters in 90 weeks old laying hens.
\end{abstract}

Keywords: calcium; laying hens; particle size. 


\section{Introducción}

La avicultura es importante en la generación de alimentos de buena calidad y bajo costo para el ser humano, y, en particular, el huevo de gallina adquiere gran protagonismo; es por ello que existen numerosos estudios destinados a determinar las necesidades de minerales, en especial de calcio, en las gallinas ponedoras. Desde el punto de vista económico, la avicultura se ve afectada por cuantiosas pérdidas debidas, entre otras cosas, a la fragilidad del cascarón, que al final aumenta el porcentaje de huevo roto.

Teniendo en cuenta que el nivel óptimo biológico y económico de calcio para gallinas ponedoras en el segundo ciclo productivo es de 3.17 g/ave/ día (1) y que el bajo consumo de alimento provoca una ingestión de calcio inferior a la necesaria para la calcificación del huevo, si se ajusta el nivel energético de la dieta en épocas de calor habrá de corregirse el nivel de calcio de esta para conseguir la ingesta adecuada. Por lo tanto, en épocas de bajo consumo de alimento, incrementar el nivel de calcio de la ración de las aves puede disminuir la palatabilidad de este.

Varios autores aconsejan el empleo de partículas groseras de carbonato cálcico, ya que mejoran la ingesta de calcio, incluso trabajando con dietas bajas en calcio; sin embargo, todavía no se conoce en realidad cuál sería el mejor tamaño de partícula de calcio para incluirlo en las raciones comerciales $(2,3)$.

Con el propósito de evaluar y comparar el efecto de la granulometría de calcio en gallinas ponedoras, en relación con la calidad del huevo, en términos del grosor y el peso de la cáscara, de las unidades Haugh, de la densidad y del pH de la albúmina y la yema, se experimentaron cuatro tratamientos con diferentes granulometrías de calcio.

\section{Materiales y métodos}

El experimento fue realizado en la granja experimental de la Facultad de Medicina Veterinaria y Zootecnia de la Universidad Cooperativa de Colombia, a $03^{\circ} 24^{\prime \prime \prime} \mathrm{N} 74^{\circ} 56^{\prime \prime \prime} \mathrm{O}$, con un rango tér- mico entre 19 y $31^{\circ} \mathrm{C}$, a una altura de $1285 \mathrm{msnm}$ y humedad relativa promedio de $70 \%$. Durante 39 días, divididos en tres ciclos de 13 días, se sometieron 120 gallinas ponedoras de la línea Hy-Line W-36, con 90 semanas de edad, alojadas en un galpón de jaulas individuales, con bebederos automáticos por jaula y un comedero por cada 3 jaulas.

Las aves fueron distribuidas con un diseño completamente al azar, con 4 tratamientos y 10 repeticiones de 3 aves en cada unidad experimental. Los tratamientos del experimento consistieron en incluir en la dieta calcio con diferentes tamaños de partícula: $\mathrm{T} 1=$ Fino $($ menor de $1 \mathrm{~mm}), \mathrm{T} 2=$ Especial $(3 \mathrm{~mm}), \mathrm{T} 3=$ Malla $(6 \mathrm{~mm})$ y T4 $=$ Piedrilla $(9 \mathrm{~mm})$.

Los huevos fueron colectados diariamente, para determinar el porcentaje de postura, y pesados individualmente en balanza de precisión (OHAUS, SCOUT-PRO $\left.{ }^{\circledR}\right)$; posteriormente, se analizaron 5 huevos por tratamiento, para determinar las variables de la calidad de huevo. En los cuatro últimos días de cada ciclo se colectaron las sobras de los comederos para calcular el consumo diario de ración.

El test de gravedad específica se realizó inmediatamente después de la colecta de los huevos, mediante inmersión de ellos en diferentes soluciones salinas, con densidades de 1.070, 1.075, 1.080, 1.085 y $1.090 \mathrm{~g} / \mathrm{ml}$. El hidrómetro flota primero en un líquido de referencia (agua), y el desplazamiento del agua se marca en el tallo; luego, al ponerlo en un líquido con nueva densidad, flota y marca la gravedad específica a través de la diferencia en el desplazamiento.

Enseguida, los huevos fueron abiertos y puestos en una superficie de vidrio horizontal para medir, con micrómetro digital Mitutoyo ${ }^{\circledR}$, la altura de la albúmina densa y de la yema. El pH de albúmina y yema se midió introduciendo en estas un pHmetro portátil Schott ${ }^{\circledR}$.

La calidad interna del huevo fue medida por la Unidad Haugh, calculada con la siguiente fórmula: $\mathrm{UH}=100 \log (\mathrm{h}-1.7 \mathrm{p} 0.37+7.6)$, donde $\mathrm{UH}=\mathrm{Uni}-$ 
dad Haugh, $\mathrm{h}=$ Altura de la albumina densa $(\mathrm{mm})$ y $\mathrm{p}=$ Peso del huevo (g).

Las cáscaras fueron lavadas y secadas a temperatura ambiente durante 48 horas, $y$, enseguida, fue calculado el porcentaje de cáscara con relación al peso del huevo. Posteriormente, se midió la espesura de las cáscaras mediante un micrómetro manual Wembley ${ }^{\circledR}$.

Una vez se confirmaron los supuestos estadísticos, los datos fueron sometidos a ANOVA, adoptándose el $5 \%$ de probabilidad para determinar dife- rencias significativas entre grupos; posteriormente, se realizó un test pos Hoc de Tukey, utilizando el software estadístico INFOSTAT.

\section{Resultados}

En la Tabla I se observa que el consumo de alimento y la conversión alimenticia fue menor en el T4 $(\mathrm{P}<0.05)$, mientras que el peso del huevo no se vio afectado $(p>0,05)$ y el porcentaje de postura fue significativamente mayor en los tratamientos T2 y T3 $(p<0,05)$.

Tabla I. Consumo de alimento (g), Peso del huevo (g), Conversión alimenticia $(\mathrm{kg} / \mathrm{kg}$ ) y Porcentaje de postura en gallinas ponedoras alimentadas con diferentes granulometrías de calcio.

\begin{tabular}{|c|c|c|c|c|c|c|c|}
\hline \multirow{3}{*}{ Variable } & \multicolumn{4}{|c|}{ Tratamientos } & \multirow[b]{3}{*}{ EEM } & \multirow[b]{3}{*}{$\mathrm{CV}$} & \multirow[b]{3}{*}{ P Valor } \\
\hline & T1 & $\mathrm{T} 2$ & T3 & T4 & & & \\
\hline & $1 \mathrm{~mm}$ & $3 \mathrm{~mm}$ & $6 \mathrm{~mm}$ & $9 \mathrm{~mm}$ & & & \\
\hline Consumo $^{1}$ & $90.6^{a}$ & $91.9^{a}$ & $92.2^{\mathrm{a}}$ & $80.1^{\mathrm{b}}$ & 1.49 & 9.2 & $0.001^{*}$ \\
\hline Peso de huevo & 66.05 & 66.26 & 66.73 & 68.43 & 0.69 & 5.7 & 0.0679 \\
\hline$C A^{2}$ & $1.37^{b}$ & $1.39^{b}$ & $1.39^{b}$ & $1.17^{\mathrm{a}}$ & 0.03 & 10.4 & $0.001^{*}$ \\
\hline$\%$ de postura ${ }^{3}$ & $54.1^{\mathrm{ab}}$ & $57.97^{\mathrm{a}}$ & $60.54^{a}$ & $49.9^{b}$ & 2.13 & 21.01 & $0.0038 *$ \\
\hline
\end{tabular}

En la Tabla II se evidencia que las diferentes granulometrías de calcio no afectaron significativamente de la albúmina, el $\mathrm{pH}$ de la yema ni las Unidades Haugh ( $p>0.05)$, y que la densidad del huevo auel peso de la cáscara, el espesor de la cáscara, la mentó significativamente. altura de la albúmina, la altura de la yema, el pH

Tabla II. Densidad $\left(\mathrm{g} / \mathrm{cm}^{3}\right)$, Peso cáscara (g), Espesor de la cáscara (micras), Altura de albúmina (mm), Altura yema (mm), pH albúmina, $\mathrm{pH}$ yema y Unidad Haugh (UH), en huevos de gallinas ponedoras alimentadas con diferentes granulometrías de calcio.

\begin{tabular}{|c|c|c|c|c|c|c|c|}
\hline \multirow{3}{*}{ Variable } & \multicolumn{4}{|c|}{ Tratamientos } & & & \multirow[b]{3}{*}{ P Valor } \\
\hline & T1 & $\mathrm{T} 2$ & T3 & T4 & & & \\
\hline & $(1 \mathrm{~mm})$ & $(3 \mathrm{~mm})$ & $(6 \mathrm{~mm})$ & $(9 \mathrm{~mm})$ & EEM & $\mathrm{CV}$ & \\
\hline Densidad & $1066.4^{b}$ & $1068.4^{\mathrm{a}}$ & $1067.4^{\mathrm{ab}}$ & $1066.9^{\mathrm{ab}}$ & 0.50 & 5.77 & $0.0357^{*}$ \\
\hline Peso cáscara & 5.67 & 5.60 & 5.59 & 5.77 & 0.12 & 16.00 & 0.6839 \\
\hline Espesor cáscara & 33.93 & 34.65 & 34.51 & 35.03 & 0.39 & 8.66 & 0.2506 \\
\hline $\begin{array}{l}\text { Altura de albúmi- } \\
\text { na }\end{array}$ & 5.50 & 5.32 & 5.17 & 5.37 & 0.13 & 19.20 & 0.3148 \\
\hline Altura yema & 16.33 & 16.07 & 16.35 & 16.32 & 0.13 & 6.37 & 0.3924 \\
\hline
\end{tabular}




\begin{tabular}{|c|c|c|c|c|c|c|c|}
\hline \multirow{3}{*}{ Variable } & \multicolumn{4}{|c|}{ Tratamientos } & & & \multirow[b]{3}{*}{ P Valor } \\
\hline & T1 & T2 & T3 & T4 & & & \\
\hline & (1 mm) & $(3 \mathrm{~mm})$ & $(6 \mathrm{~mm})$ & $(9 \mathrm{~mm})$ & EEM & CV & \\
\hline pH albúmina & 7.87 & 7.92 & 8.00 & 8.01 & 0.06 & 6.13 & 0.3039 \\
\hline $\mathrm{pH}$ yema & 6.22 & 6.11 & 6.12 & 6.09 & 0.04 & 5.16 & 0.1264 \\
\hline UH & 69.75 & 68.81 & 66.19 & 67.09 & 1.19 & 13.53 & 0.1839 \\
\hline
\end{tabular}

EEM: Error estándar de la media, CV: Coeficiente de variación. * Letras diferentes indican diferencias significativas $(p<0,05)$.

\section{Discusión}

Según Etches (4), existen 3 fuerzas que regulan el consumo de calcio en aves de postura: el ritmo circadiano, la secuencia ovulatoria y el método de presentación de este mineral para su consumo. Este último parámetro ha sido demostrado mediante estudios comparativos entre carbonato de calcio en polvo y granulado en dietas de gallinas ponedoras de segundo ciclo productivo, en los cuales se reporta que en el carbonato de calcio granulado mejora la calidad de la cáscara y la gravedad específica del huevo (3). Estos reportes confirman la teoría de quienes afirman que las partículas de mayor tamaño de calcio proporcionan mayor retención en la parte superior del tracto digestivo (5), haciendo que el calcio esté disponible, lenta y uniformemente, durante el período de formación de la cáscara del huevo.

Los resultados obtenidos en el presente experimento demuestran que las gallinas ponedoras mejoran el consumo de alimento cuando la dieta tiene partículas de carbonato de calcio hasta de 6 $\mathrm{mm}$, mientras que las dietas que contienen partículas de carbonato de calcio de $9 \mathrm{~mm}$, aunque no afectan la calidad del huevo, si afectan negativamente el consumo de alimento. En consecuencia, la deficiencia de calcio y demás nutrientes en gaIlinas ponedoras se ve reflejada en la disminución del peso del huevo y del porcentaje de postura, de la misma manera como se afecta en codornices (6).

Al finalizar el experimento de la presente investigación, se realizó necropsia a 5 gallinas de cada tratamiento, y se reportó que en las aves del T1 no hubo hallazgo de partículas de carbonato de calcio almacenadas en la molleja, a diferencia de los demás tratamientos, en los que sí se encontraron partículas remanentes en la molleja, al igual que en los reportes de publicaciones previas (7). Por lo anterior, se infiere que las gallinas alimentadas con carbonato de calcio de tamaño entre 3 y $9 \mathrm{~mm}$ optimizan la disponibilidad de calcio en el tracto digestivo para el momento de la formación de la cáscara, aunque los resultados de productividad permiten concluir que la granulometría óptima para el uso en gallinas ponedoras de segundo ciclo productivo es máximo de $6 \mathrm{~mm}$. No obstante, lo anterior, es necesario evaluar la movilización total de calcio a partir de los huesos y la resistencia a la ruptura de la tibia, con propósitos de evaluar el bienestar en las gallinas (3).

Concordante con hallazgos previos (8), se obtuvieron mejores rendimientos de postura y consumo de alimento con las dietas que contienen partículas más finas de calcio. Sin embargo, a pesar de que el porcentaje de postura y el consumo de alimento es menor en el tratamiento 4, que contiene las partículas más gruesas de carbonato de calcio, la conversión alimenticia y el peso del huevo son significativamente mejores, comparados con los demás tratamientos, lo cual debe evaluarse en un estudio financiero de acuerdo con los procesos de mercadeo llevados a cabo en el sistema productivo.

Teniendo en cuenta que los requerimientos de calcio y fósforo dependen de la edad de las aves, de tal manera que en el segundo ciclo productivo se requiere una suplementación de $4.5 \%$ de calcio y $0,25 \%$ de fósforo disponible para mejorar la conversión alimenticia y el grosor de la cáscara (9), se hace necesario evaluar el efecto de la granulometría de calcio sobre los requerimientos de fósforo. 
Otro aspecto que es necesario evaluar es la procedencia del calcio, puesto que se han reportado diferencias significativas entre el que proviene de piedra caliza y el proveniente de concha de ostras, en parámetros productivos comparados, pero no sobre la calidad del huevo ni el grosor de la cáscara (2); sin embargo, los autores no especifican la granulometría de los productos.

Excepto la densidad de los huevos, los demás parámetros de calidad de huevo evaluados en este experimento no se vieron afectados por la granulometría del carbonato de calcio, manteniendo los datos en el promedio reportado por la literatura en el segundo ciclo productivo $(10,11)$.

\section{Conclusiones}

En dietas de gallinas ponedoras de 90 semanas de edad es posible incluir carbonato de calcio de 3 y $6 \mathrm{~mm}$ de diámetro, con el fin de mejorar porcentaje de postura y de consumo de alimento.

\section{Agradecimientos}

Los autores agradecen a la Universidad Cooperativa de Colombia, sede Ibagué, por su apoyo logístico y financiero.

\section{Referencias}

(1) Hernández-Sánchez J, Cuca-García M, Pró-Martínez A, González-Alcorta M, Becerril-Pérez $\mathrm{C}$. Nivel óptimo biológico y económico de calcio en gallinas Leghorn blancas de Segundo ciclo de postura. Agrociencia. 2006; 40: 49-57.

(2) Ahmed N, Abdel A, Elamin K, Dafalla K, Malik $H$, Dousa B. Effect of dietary calcium sources on laying hens performance and egg quality. J Anim Prod Adv. 2013; 3 (7): 226-231. DOI: http://dx.doi.org/10.5455/ japa.20130718034818.
(3) Cuca M, Valdes V, Pro A, Suarez M, Figueroa J, González M. Nivel de calcio y relación carbonato de calcio pulverizado: granulado y su efecto en producción de huevo y calidad del cascarón en gallinas pelechadas. Sitio Argentino de Producción Animal. 2007; 1-5.

(4) Etches R. Calcium. Logistic in the laying hen. The J. of nutr. 1987; 117(3): 619-625.

(5) Leeson. La nutrición y la calidad de la cáscara. Nutrición Aviar Comercial. 2000; 178-304.

(6) Mogyca N, Morais R, Barbosa A, Barcellos M, Stringhini J, Gonzales E. Granulometria do calcário no desempenho e qualidade da casca de ovos de codornas japonesas. Ciência Anim Bras. 2006; 7(4): 381-387.

(7) Tsuyoshi D, de Faria D, Aiko E, Junqueira O, de Araujo L. Efeitos do fracionamento do cálcio dietário e granulometria docalcário sobre o desempenho e qualidade dos ovos de ponedeiras comerciais. Acta Sci Anim Sci. 2006; 28(2): 187-195.

(8) Geraldo A. Níveis de cálcio e granulometrias do calcário para frangas de reposição no período de 3 a 12 semanas de idade. R. Bras. Zootec. 2006; 35(1): 113-118. DOI: http://dx.doi. org/10.1590/S1516-35982006000100014.

(9) Pelicia K, Garcia E, Faitarone A, Silva A, Berto D, Molino A, Vercese F. Calcium and Available Phosphorus Levels for Laying Hens in Second Production Cycle. Brazilian J Poult Sci. 2009; 11(1): 39-49. DOI: http://dx.doi. org/10.1590/s1516-635x2009000100007.

(10) Moraes E, Ramos N, Kleszcz F, Barbosa J. Granulometria do calcário: desempenho e qualidade dos ovos de poedeiras em final de produção. Zootecnia Trop. 2012; 30(4): 311-316.

(11) Cubillos. Enciclopedia de la inspección veterinaria y análisis de los alimentos. España, Espasa-Calpe. 2003; 965-990. 